\title{
Concordancia interobservador en la clasificación de Paprosky en un hospital de un país de una economía emergente
}

\author{
Inter-observer agreement in the Paprosky classification in a \\ hospital in a country of an emerging economy \\ Macedonio-García A, ${ }^{*}$ Rivera-Villa AH, ${ }^{\ddagger}$ Quiroz-Williams J, ${ }^{\S}$ Aguilera-Martínez P ${ }^{ף}$ \\ Hospital de Ortopedia de la UMAE «Dr. Victorio de la Fuente Narváez».
}

RESUMEN. Introducción: La evaluación y manejo de la pérdida ósea acetabular en la artroplastía total de cadera es un desafío para los cirujanos ortopédicos, deseamos en este estudio determinar la concordancia interobservador en la clasificación de Paprosky para defectos acetabulares. Material y métodos: Estudio observacional, descriptivo, longitudinal, prospectivo. Se realizó utilizando las radiografías anteroposteriores de cadera de pacientes programados para artroplastía total de cadera primaria o de revisión con defecto óseo acetabular, sistema de rayos X local Sinapse, se valoraron 20 radiografías por observador tomadas de manera aleatoria (médicos adscritos al servicio de reemplazos articulares con experiencia mínima de cinco años) utilizando la clasificación de Paprosky de manera espontánea (sin clases previas) de defectos acetabulares, se evaluó la concordancia interobservador mediante el coeficiente de kappa de Cohen. Resultados: Se obtuvo una concordancia interobservador en promedio para la clasificación de Paprosky de defectos acetabulares utilizando el coeficiente de kappa de Cohen, el análisis de concordancia se hizo empleando
ABSTRACT. Introduction: The evaluation and management of acetabular bone loss in total hip arthroplasty is a challenge for orthopedic surgeons, we wish in this study to determine the inter-observer concordance in the Paprosky classification for acetabular defects. Material and methods: Observational, descriptive, longitudinal, prospective study. It was performed using anteroposterior hip radiographs of patients scheduled for primary total hip arthroplasty or revision with acetabular bone defect, Sinapse local X-ray system, $20 \mathrm{X}$-rays were assessed per observer taken randomly (doctors assigned to the joint replacement service with minimum experience of five years) using the Paprosky classification spontaneously, (without previous classes) for acetabular defects, inter-observer concordance was assessed using Cohen's Kappa coefficient. Results: An interobserver agreement was obtained on average for the Paprosky classification of acetabular defects using the Cohen's Kappa coefficient, the concordance analysis was done using the statistical program statistical package for the social sciences of 0.237 . Conclusions: Both the null

\section{Nivel de evidencia: III}

\footnotetext{
* Médico Especialista en Ortopedia y Traumatología. Alta Especialidad en Reemplazos Articulares. Hospital de Ortopedia

* Médico Especialista en Traumatología y Ortopedia. Jefe del Departamento Clínico de Reemplazos Articulares.

$\S$ Médico Especialista en Traumatología y Ortopedia. Jefe del Departamento de Investigación en Salud.

๑ Médico Especialista en Traumatología y Ortopedia. Médico adscrito al Servicio de Reemplazos Articulares.
}

Unidad Médica de Alta Especialidad (UMAE) «Dr. Victorio de la Fuente Narváez».

Correspondencia:

Dr. Arturo Macedonio-García

Colector 15 s/n (Av. Fortuna), Esq. Av. Politécnico Nacional, Col. Magdalena de las Salinas, C.P. 07760, Alcaldía Gustavo A Madero, Ciudad de México.

Tel: 5747-3500, ext. 25537.

E-mail: dr.arturomacedoniogarcia@gmail.com

Recibido: 12-06-2019. Aceptado: 12-01-2021.

Citar como: Macedonio-García A, Rivera-Villa AH, Quiroz-Williams J, Aguilera-Martínez P. Concordancia interobservador en la clasificación de Paprosky en un hospital de un país de una economía emergente. Acta Ortop Mex. 2021; 35(3): 257-260. https://dx.doi.org/10.35366/102363 
el programa estadístico Statistical Package for the Social Sciences de 0.237. Conclusiones: Tanto la hipótesis nula (kappa mayor de 0.80 valor considerado óptimo) como la hipótesis alternativa (kappa de 0.41 a 0.80 valor igual a moderada a buena) no concordaron con nuestro estudio (kappa igual a 0.237 ) valor igual a leve, además se demostró que a mayor defecto acetabular menor es la concordancia interobservador en cirujanos en nuestra institución.

Palabras clave: Paprosky, defecto acetabular, concordancia, prótesis de cadera. hypothesis (Kappa greater than 0.80 value considered optimal) and the alternative hypothesis (Kappa from 0.41 to 0.80 value equal to moderate to good) did not agree with our study (Kappa equal to 0.237 ) value equal to mild, in addition, it was shown that the greater the acetabular defect, the lower the interobserver agreement in surgeons in our institution.

Keywords: Paprosky, acetabular defect, concordance, hip prosthesis.

\section{Introducción}

Las expectativas del aumento de la demanda de la artroplastía total de cadera primaria durante las próximas décadas aseguran el incremento de las artroplastías totales de cadera de revisión. ${ }^{1}$ Se proyecta que las revisiones aumenten de 40,800 casos en 2005 a 96,700 casos en $2030 .^{2} \mathrm{La}$ revisión del componente acetabular contabiliza para más de $50 \%$ de los casos y las causas comunes de estos procedimientos son inestabilidad, infección, desgaste de polietileno y aflojamiento aséptico. ${ }^{3}$ La pérdida ósea severa resulta de la osteólisis asintomática, stress shielding y migración del componente. ${ }^{4}$ Uno de los aspectos más desafiantes de la artroplastía total de cadera primaria y de revisión es el manejo de la pérdida ósea acetabular que compromete la estabilidad y la fijación del implante, una situación que se volverá más común en un futuro cercano. La clasificación de Paprosky descrita por primera vez en 1994 es una de las clasificaciones de defectos acetabulares más ampliamente utilizada. ${ }^{5}$

Se han diseñado múltiples sistemas de clasificación para describir los defectos óseos acetabulares previamente a la cirugía: Paprosky, ${ }^{6}$ D'Antonio ${ }^{7}$ o American Academy of Orthopaedic Surgeons (AAOS); Saleh, ${ }^{8}$ Gustilo, ${ }^{9}$ Bruto, ${ }^{10}$ Parry ${ }^{11}$ y Engh por mencionar sólo algunas, siendo la más utilizada en nuestra unidad la clasificación de Paprosky.

Los defectos se clasifican de acuerdo a la presencia o ausencia de paredes acetabulares intactas y la capacidad de las columnas anterior y posterior para soportar un implante; los hallazgos preoperatorios en la radiografía anteroposterior de la pelvis se usan para calcular el tipo de defecto y la planificación de la técnica reconstructiva adecuada; hay cuatro criterios para evaluarla: migración superior del centro de la cadera, osteólisis del isquion, osteólisis de la lágrima y posición del implante en relación a la línea de Kohler.

Existen tres tipos de defectos acetabulares básicamente según Paprosky: los defectos tipo 1 tienen sólo un mínimo de pérdida ósea, ausencia de migración de los componentes y paredes acetabulares intactas. Los defectos de tipo 2 tienen una pérdida ósea moderada con distorsión del hemisferio acetabular a excepción de la preservación de las columnas acetabulares anterior y posterior. La destrucción involucra a los muros superiores y/o medial y se basa en su ubicación, los defectos de tipo 2 se subclasifican en $2 \mathrm{~A}$ (afectación superolateral), 2B (superomedial) y 2C (medial). Los defectos tipo 3 muestran una pérdida ósea severa, destrucción mayor del borde acetabular y las estructuras de soporte; estos son subclasificados en 3A y 3B. Los defectos tipo 3A incluyen destrucción moderada de la pared medial y columna posterior, mientras que los defectos tipo 3B muestran destrucción completa de la pared medial y destrucción severa de la columna posterior. Basado en el tipo de defecto acetabular, Paprosky determinó un algoritmo de tratamiento. . $^{5,12}$

A pesar de su popularidad mundial, en nuestro hospital sigue habiendo disputa sobre la confiabilidad y la validez de la clasificación de Paprosky, ya que para los médicos con poca familiaridad con la clasificación se presenta de manera compleja el poder determinar el tipo de defecto acetabular y hay discrepancia entre los mismos, además un análisis estructurado podría mejorar su confiabilidad interobservador. ${ }^{13}$ En nuestra institución, IMSS y en México no hay estudios que valoren la concordancia interobservador y los que se han realizado fueron hechos en Canadá, Estados Unidos y Australia reportando una concordancia de moderada a buena para esta clasificación. ${ }^{13,14,15}$

La clasificación apropiada del defecto acetabular preoperatorio sigue siendo discutible. Un sistema válido de clasificación preoperatoria confiable puede ayudar en la planificación preoperatoria efectiva y permitir así una mejora en el tratamiento evitando eventualidades transquirúrgicas y posquirúrgicas. ${ }^{16,17}$

\section{Material y métodos}

Tipo de estudio observacional, descriptivo, longitudinal, prospectivo. El universo de trabajo se realizó utilizando las radiografías preoperatorias anteroposteriores en pacientes programados para artroplastía total primaria o de revisión de cadera, se valoró un total de 20 radiografías de la plataforma «SINAPSE» del Departamento de rayos $\mathrm{X}$ del HOVFN por médicos dedicados al manejo del reemplazo articular de cadera adscritos al Servicio de Endoprótesis del Departamento de Reemplazos Articulares con una experiencia mínima de cinco años haciendo reemplazo de cadera primaria y de revisión. 
1. Se invitó al médico, previa descripción del estudio y firma de consentimiento informado.

2. Se aplicó el cuestionario mostrando las 20 imágenes radiográficas anteroposteriores de pacientes programados a reemplazo articular de cadera primaria o de revisión tomadas del sistema virtual SINAPSE, dichas imágenes fueron clasificadas de acuerdo a la clasificación de Paprosky por un monitor experto en el tema; se mostraron en una tablet sin previo aviso de la fecha en que se les realizaría el test, anotando sus observaciones cada médico en el formato especíico.

3. Se valoró el grado de concordancia interobservador.

4. Análisis de concordancia.

Para determinar la concordancia interobservador se utilizó la prueba estadística kappa de Cohen, que es la indicada para evaluar la concordancia entre dos observadores en variables nominales dicotómicas. Se evaluó la concordancia absoluta con un intervalo de confianza (IC) al $95 \%$. La interpretación del coeficiente kappa se realizó al correlacionar su valor con una escala cualitativa que incluye seis niveles de fuerza de concordancia: «pobre» $(\kappa=0)$, «leve» $(\kappa=0.01-0.2)$, «aceptable» $(\kappa=0.21-0.4)$, «moderada» $(\kappa$ $=0.42-0.6)$, «buena o considerable» $(\kappa=0.61-0.8)$ y «casi perfecta» $(\kappa=0.81-1) \cdot{ }^{17,18}$

El coeficiente de kappa se usa para evaluar la concordancia o reproducibilidad de instrumentos de medida cuyo resultado es categórico (dos o más categorías). En este estudio no se calcula el tamaño de la muestra por el tipo de variable a determinar. Una vez obtenido el coeficiente de kappa de Cohen mediante el programa SPSS se tiene el grado de acuerdo a la escala Sackett D y colaboradores.

\section{Resultados}

Participó un total de seis médicos que reunieron los criterios de inclusión. Se realizó un análisis con el programa SPSS (Statistical Package for the Social Sciences) previo al archivo de los datos en programa Excel.

Para obtener el resultado de la concordancia interobservador se utilizó la valoración radiográfica por parte de los especialistas de 20 radiografías con patología acetabular elegidas aleatoriamente.

Los resultados por observador se agruparon en la Tabla 1 , obteniéndose una concordancia interobservador en pro-

\begin{tabular}{|ccc|}
\hline \multicolumn{3}{|c|}{ Tabla 1: Resultados por observador. } \\
\hline Observador & $\begin{array}{c}\text { Resultado kappa } \\
\text { de Cohen }\end{array}$ & $\begin{array}{c}\text { Grado } \\
\text { de acuerdo }\end{array}$ \\
\hline 1 & 0.203 & Pobre \\
2 & 0.062 & Pobre \\
3 & 0.169 & Pobre \\
4 & 0.430 & Moderado \\
5 & 0.315 & Leve \\
6 & 0.245 & Pobre \\
& Promedio & Kappa $=\mathbf{0 . 2 3 7}$ \\
\end{tabular}

Tabla 2: Nivel de acuerdo según Sackett $D$, et al. ${ }^{18}$

\begin{tabular}{|cc|}
\hline Valor de Kappa & Grado de acuerdo \\
\hline 0 & Ninguno \\
$0.01-0.20$ & Pobre \\
$0.21-0.40$ & Leve \\
$0.41-0.60$ & Moderado \\
$0.61-0.80$ & Bueno \\
$0.81-0.99$ & Óptimo \\
\hline
\end{tabular}

medio para la clasificación de Paprosky de defectos acetabulares de 0.237 , lo cual equivale a un grado leve según Sackett (Tabla 2). ${ }^{18}$

\section{Discusión}

La clasificación apropiada para valorar los defectos acetabulares preoperatorios sigue siendo discutible. ${ }^{17,19,20,21}$ Un sistema de clasificación válido y confiable puede ayudar en una planificación preoperatoria efectiva, permitir comparaciones y hacerse mediante diferentes técnicas. ${ }^{14}$ La clasificación original de Paprosky se basa en el análisis de radiografías AP de la pelvis y por lo tanto, intenta clasificar estructuras tridimensionales complejas usando una imagen bidimensional. Áreas pequeñas o la lisis no se aprecian bien en las radiografías, la clasificación ahora se ha perfeccionado con cuatro parámetros radiográficos específicos que proporcionan criterios para clasificar cada defecto con precisión aparentemente. ${ }^{20}$ Varios estudios se han realizado para determinar la confianza interobservador así como la validez de dicha clasificación obteniendo resultados que van de moderados a buenos (coeficiente de kappa de Cohen de 0.41 a 0.80 ). . $^{11,13,15,16}$

Este estudio tiene varias limitaciones, hay una limitación en el grado de información obtenida de una comparación entre datos categóricos y cuantitativos; sin embargo, es evidente que los defectos radiográficos de mayor grado, según la clasificación de Paprosky, están asociados con mayor superficie de defectos de área en las paredes acetabular anterior, medial y superior; de igual modo para valorar la región posterior se requiere el uso de radiografías (rx) oblicuas de entrada y salida pélvica; sin embargo, la clasificación así lo dictamina, usando sólo radiografías AP; además, ninguno de los médicos tuvo sesiones académicas previas al estudio con respecto al uso de esta clasificación, dado que la conocían y aplicaban rutinariamente.

En México no se encuentran estudios de concordancia interobservador que valoren esta clasificación, motivo por el cual se decidió realizar este estudio, además los resultados obtenidos no se habían reportado en la literatura, ya que encontramos una concordancia de coeficiente de kappa de Cohen de 0.237 siendo ésta un grado de concordancia leve según Sackett, ${ }^{18}$ por lo cual consideramos sumamente importante el desarrollo de una clasificación más sencilla para mejorar la valoración preoperatoria de esta patología así como los resultados transquirúrgicos y postquirúrgicos en 
beneficio de los pacientes y que como toda buena clasificación debe ser diagnóstica, pronóstica y terapéutica. Ya en otros estudios se ha mencionado que la capacitación con varias sesiones académicas (a partir de una a tres sesiones) es necesaria para poder emplear de mejor manera la clasificación de Paprosky; sin embargo, el resultado de la concordancia sigue siendo el mismo, incluso se menciona que el uso de la tomografía puede ser muy útil, como lo es en las fracturas acetabulares, para realizar una mejor planeación preoperatoria. ${ }^{15} \mathrm{La}$ discordancia observada podría disminuir quizás con la definición de parámetros más claros para evaluar la presencia de algún hallazgo. Esto se podría conseguir con criterios objetivos basados en mediciones comparativas o proporcionales, ya que se consideró que no es factible realizar una planeación de una estructura anatómica tan compleja con un estudio que valora sólo en dos planos (radiografías AP y lateral, u oblicuas), siendo el acetábulo una estructura tridimensional sería mejor utilizar una tomografía computarizada con reconstrucción tridimensional para una mejor planeación quirúrgica.

Otro factor importante a señalar es que a mayor grado de lesión y experiencia del cirujano menor es la concordancia, como lo mencionan Campbell y colaboradores, ${ }^{16}$ quienes informaron que incluso Paprosky encontró sólo un buen resultado, destacando aún más la dificultad de analizar la pérdida de hueso acetabular por alguien máximamente familiarizado con el esquema de clasificación.

Este estudio servirá de base para el diseño y posterior evaluación estadística de nuevas estrategias para la definición objetiva al evaluar los defectos óseos en la patología acetabular en beneficio de los pacientes de nuestra institución.

\section{Conclusiones}

Tanto la hipótesis nula (Kappa mayor de 0.80 valor considerado óptimo) como la hipótesis alternativa (Kappa de 0.41 a 0.80 valor igual a moderada a buena) no concordaron con nuestro estudio, ya que el resultado fue Kappa igual a 0.237 valor igual a leve.

\section{Agradecimientos}

El equipo de este trabajo agradece a las autoridades de la UMAE «Victorio de la Fuente Narváez» así como al servicio de reemplazos articulares.

Referencias

1. Deirmengianreali GK, Zmitowski B, O'Neil JT, Hozack WJ. Management of acetabular bone loss in revision total hip arthroplasty. J Bone Joint Surg Am. 2011; 93(19): 1842-52.
2. Kurtz S, Ong K, Lau E, et al. Proyections of primary and revision hip and knee arthroplasty in the United States from de 2005 to 2030. J Bone Joint Surg Am. 2007; 89(4): 780-5.

3. Bozic KJ, Kurtz SM, Lau E, et al. The epidemiology of revision total hip arthroplasty in the United States. J Bone Joint Surg Am. 2009; 91: 128-33.

4. Sporer SM, Paprosky WG, O'Rourke MR. Managing bone loss in acetabular revision. Instr Course Lect. 2006; 55: 287-97.

5. Paprosky WG, Bradford MS, Younger TI. Classification of bone defects in failed prostheses. Chir Organi Mov. 1994; 79(4): 285-91.

6. Paprosky WG, Perona PG, Lawrence JM. Acetabular defect classification and surgical reconstruction in revision arthroplasty. A 6-year follow-up evaluation. J Arthroplasty. 1994; 9: 33-44.

7. D'Antonio JA. Periprosthetic bone loss of the acetabulum: classification and management. Orthop Clin North Am. 1992; 23: 279-90.

8. Saleh KJ, Holtzman J, Gafni A, Saleh L, Jaroszynski G, Wong P, Woodgate I, Davis A, Gross AE. Development, test reliability and validation of a classification for revision hip arthroplasty. J Orthop Res. 2001; 19: 50-6.

9. Gustilo RB, Pasternak HS. Revision total hip arthroplasty with titanium ingrowth prosthesis and bone grafting for failed cemented femoral component loosening. Clin Orthop Relat Res. 1988; 235: 111-9.

10. Gross AE, Duncan CP, Garbuz D, Mohamed EM. Revision arthroplasty of the acetabulum in association with loss of bone stock. Instr Course Lect. 1999; 48: 57-66.

11. Parry MC, Whitehouse MR, Mehendale SA, et al. A comparison of the validity and reliability of established bone stock loss classification systems and the proposal of a novel classification system. Hip Int. 2010; 20: 50-5.

12. O'Rourke MR, Paprosky WG, Rosenberg AG. Use of structural allografts in acetabular revision surgery. Clin Orthop Relat Res. 2004; 420: 113-21.

13. Gozzard C, Blom A, Taylor A, Smith E, Learmonth I. A comparison of the reliability and validity of bone stock loss classification systems used for revision hip surgery. J Arthroplasty. 2003; 18(5): 638-42.

14. Garbuz DS, Masri BA, Esdaile J, Duncan CP. Classification systems in orthopaedics. J Am Acad Orthop Surg. 2002; 10: 290-7.

15. Yu R, Hofstaetter JG, Sullivan T, et al. Validity and reliability of the Paprosky acetabular defect classification. Clin Orthop Relat Res. 2013; 471(7): 2259-65.

16. Campbell DG, Garbuz DS, Masri BA, Duncan CP. Reliability of acetabular bone defect classification systems in revision total hip arthroplasty. J Arthroplasty. 2001; 16: 83-6.

17. Davis AM, Schemitsch EH, Gollish JD, et al. Classifying failed hip arthroplasty: generalizability of reliability and validity. Clin Orthop Relat Res. 2003; 415: 171-9.

18. Sackett DL, Haynes BR, Tugwell P, Guyatt GH. Clinical epidemiology: a basic science for clinical medicine. $2^{\text {nd }}$ ed. Boston: Brown and Company, 1991.

19. Masri BA, Masterson EL, Duncan CP. The classification and radiographic evaluation of bone loss in revision hip arthroplasty. Orthop Clin North Am. 1998; 29(2): 219-27.

20. Telleria JJM, Gee AO. Paprosky classification of acetabular bone loss. Clin Orthop Relat Res. 2013; 471(119: 3725-30.

21. Johanson NA, Driftmier KR, Cerynik DL, Stehman CC. Grading acetabular defects: the need for a universal and valid system. $J$ Arthroplasty. 2010; 25(3): 425-31.

Conflicto de intereses: Los autores declaran que no hay ningún conflicto de interés. 